\title{
Stochastic Linear Quadratic Optimal Control with Indefinite Control Weights and Constraint for Discrete-Time Systems
}

\author{
Xikui Liu, Guiling Li, and Yan Li \\ College of Mathematics and System Science, Shandong University of Science and Technology, Qingdao, Shandong 266510, China \\ Correspondence should be addressed to Yan Li; liyan@sdkd.net.cn
}

Received 6 May 2014; Accepted 9 September 2014

Academic Editor: Weihai Zhang

Copyright ( 2015 Xikui Liu et al. This is an open access article distributed under the Creative Commons Attribution License, which permits unrestricted use, distribution, and reproduction in any medium, provided the original work is properly cited.

\begin{abstract}
The Karush-Kuhn-Tucker (KKT) theorem is used to study stochastic linear quadratic optimal control with terminal constraint for discrete-time systems, allowing the control weighting matrices in the cost to be indefinite. A generalized difference Riccati equation is derived, which is different from those without constraint case. It is proved that the well-posedness and the attainability of stochastic linear quadratic optimal control problem are equivalent. Moreover, an optimal control can be denoted by the solution of the generalized difference Riccati equation.
\end{abstract}

\section{Introduction}

The linear quadratic (LQ) optimal control problem has been pioneered by Kalman [1] for deterministic systems; it is an assumption that the control weighting matrix in the cost is strictly definite. The definite LQ control problem has been investigated extensively by many researchers [2, 3]. The optimal control for the definite LQ problem has a feedback given by the solution of the Riccati equation. The extension of deterministic LQ problem to stochastic case has been playing an important role in engineering design and applications; see monographs [4-7]. Stochastic LQ control problem for the Itô systems is initiated by Wonham [4], while the nonlinear regulator problem is discussed in [8] and has caused a sequence of works [9-11]. Some of the works on this subject reveal that, for stochastic Itô systems, even if the state and control weighting matrices $Q$ and $R$ are indefinite, the corresponding stochastic LQ problem may be still well posed, which is first found in [12].

For the discrete-time LQ control problems with control and/or state dependent noises, there have been some works in literature $[13,14]$. It is worth noting that the state weight matrix is nonnegative and the control weight matrix is positive definite in both papers. However, the control weighting matrix is not required to be positive definite, or even negative [15-18]. In addition, most previous researchers mainly study indefinite stochastic LQ problems without constraints. In fact, some constraints are of considerable importance in many physical systems. The finite time indefinite stochastic LQ control with linear terminal state constraint is discussed in [19] and has been extended in [20-22]. It is generally known that, for the system components are perturbed by an additive Gaussian white noise, the LQ problem is called linear quadratic Gaussian problem. As said in [15], many real systems are not only subject to Gaussian white noise, but also subject to non-Gaussian noise.

In this paper, different from [20-22], we discuss a stochastic optimal control of discrete-time systems which are subject to non-Gaussian noises. We concentrate our attention on the finite horizon indefinite stochastic LQ control with terminal inequality constraint. Such constraints are often seen in $H_{\infty}$ filtering problems $[23,24]$. The existence of optimal linear state feedback control in terms of KKT theorem will be shown. We present the fact that the solvability of the GDRE, the well-posedness, and the attainability of the LQ problem are all equivalent. The outline of this paper is organized as follows. In Section 2, we give some definitions and preliminaries. Section 3 contains our main theorems. A necessary condition for the existence of optimal linear state feedback control is derived. Moreover, it is shown that the solvability of the GDRE, the well-posedness, and the attainability of the LQ problem are all equivalent. 
In Section 4, we give the structure of the optimal control. Section 5 concludes the paper.

For convenience, we adopt the following notations in this note. $M^{\prime}$ : is the transpose of a matrix $M ; \operatorname{tr}(M)$ is the trace of a square matrix $M ; M>0(M \geq 0): M$ is positive definite (positive semidefinite) symmetric matrix; $E[x]$ represents the mathematical expectation of a random variable $x ; R^{k}$ is the $k$-dimensional Euclidean space with the usual 2-norm $\|\cdot\|$; $R^{m \times n}$ is the vector space of all $m \times n$ matrices with entries in $R ; M^{\dagger}$ is the Moore-Penrose pseudoinverse of a matrix $M ; I$ is the identity matrix with appropriate dimension; $N_{t}$ : $\{0,1,2, \ldots, t-1\}$.

\section{Preliminaries}

Consider the discrete-time stochastic system $\Sigma^{*}$ :

$$
\begin{gathered}
x(k+1)=\left[A(k)+\omega^{x}(k) C(k)\right] x(k) \\
+\left[B(k)+\omega^{u}(k) D(k)\right] u(k)+\omega(k), \\
k \in N_{T}, \\
a_{i 1} x_{1}(T)+a_{i 2} x_{2}(T)+\cdots+a_{i n} x_{n}(T) \leq \xi_{i},
\end{gathered}
$$

where $x(0)=x_{0} \in R^{n}$ is the given initial state and $x(k) \epsilon$ $R^{n}$ and $u(k) \in R^{m}$ are, respectively, the system state and controlled input. $A(k), C(k) \in R^{n}, B(k)$, and $D(k) \in R^{n \times m}$ are matrix-valued functions with appropriate dimensions.

The noises $\omega^{x}(k), \omega^{u}(k), \omega(k)$ are defined on a complete probability space $\{\Omega, F, \mu\}$. Without loss of generality, we assume that $\omega^{x}(k), \omega^{u}(k)$ are scalar random variables. The initial state is assumed to be independent of $\omega^{x}(k), \omega^{u}(k), \omega(k)$ and satisfies $E\left[\omega^{x}(k)\right]=E\left[\omega^{u}(k)\right]=E[\omega(k)]=$ $0, E\left[\left(\omega^{x}(k)\right)^{2}\right]=E\left[\left(\omega^{u}(k)\right)^{2}\right]=1, E\left[\omega(k) \omega^{\prime}(k)\right]=$ $V(k), E\left[\omega^{x}(k) \omega^{u}(k)\right]=\rho^{x u}(k)$, and $E\left[\omega(k) \omega^{x}(k)\right]=$ $E\left[\omega(k) \omega^{u}(k)\right]=0$.

We denote $F_{k}$ the $\sigma$-algebra generated by $\left(\omega^{x}(k), \omega^{u}(k), \omega(k)\right)$; that is, $F_{k}=\left\{\sigma\left(\omega^{x}(k), \omega^{u}(k), \omega(k)\right.\right.$ : $\left.\left.k \in N_{T}\right)\right\} . u(\cdot)$ belongs to the admissible control set $U_{\mathrm{ad}}=\left\{u(k) \in R^{m}: E\left[\sum_{k=0}^{T-1}|u(k)|^{2}\right]<+\infty\right\} . \xi_{i}$ is $F_{T}$ measurable square integrable stochastic process; namely, $E\left[\left|\xi_{i}\right|\right]<+\infty$. Let $N_{r \times n}=\left(a_{i j}\right)_{r \times n}, \xi=\left(\xi_{1}, \xi_{2}, \ldots, \xi_{r}\right)^{\prime}$; then the constraint in (1) can be denoted by $N x(T) \leq \xi$, where $a_{i j}$ is constant and $N$ has row full rank.

We consider the following cost function correlated with the system $\Sigma^{*}$ :

$$
\begin{gathered}
J\left(x_{0}, u(0), \ldots, u(T-1)\right) \\
=\sum_{k=0}^{T-1} E\left[\left(x^{\prime}(k) Q(k) x(k)+u^{\prime}(k) R(k) u(k)\right)\right. \\
\left.+x^{\prime}(T) S x(T)\right]
\end{gathered}
$$

where $Q(k), S$, and $R(k)$ are symmetric matrices with appropriate dimension, which are possibly indefinite. We define

$$
V\left(x_{0}\right)=\inf _{u(0), \ldots, u(T-1)} J\left(x_{0}, u(0), \ldots, u(T-1)\right) .
$$

In the sequel, we study the LQ problem for the systems (1)-(3), that is to say, finding a control to minimize $J\left(x_{0}, u(0), \ldots, u(T-1)\right)$. Firstly, we state some useful definitions and lemmas that are essential to the discussions of our main results.

Definition 1. If $V\left(x_{0}\right)>-\infty$ for any $x_{0}$, systems (1)-(3) are called well posed.

Definition 2. If there exists an admissible control $\left(u_{*}(0), \ldots, u_{*}(T-1)\right)$ such that

$$
V\left(x_{0}\right)=J\left(x_{0}, u_{*}(0), \ldots, u_{*}(T-1)\right),
$$

then systems (1)-(3) are said to be attainable and $\left(u_{*}(0), \ldots, u_{*}(T-1)\right)$ is called an optimal control.

If a linear feedback control is optimal for the LQ problem (1)-(3), then it must be also optimal linear feedback control of the following form:

$$
u(k)=K(k) x(k), \quad k \in N_{T},
$$

where $K(k)$ is matrix-valued function.

MP (mathematical programming)

$$
\begin{array}{ll}
\min & f(x) \\
\text { s.t. } & \mathbf{g}(x) \leq \mathbf{0} \\
& \mathbf{h}(x)=\mathbf{0},
\end{array}
$$

where $\mathbf{g}(x)=\left(g_{1}(x), \ldots, g_{p}(x)\right), \mathbf{h}(x)=\left(h_{1}(x), \ldots, h_{q}(x)\right)$.

Definition 3 (regularity condition see [25]). Let $I^{*}=\{i \mid$ $\left.g_{i}\left(x^{*}\right)=0\right\}$. If the gradient vectors $\nabla g_{i}\left(x^{*}\right), i \in I^{*}$, and $\nabla h_{j}\left(x^{*}\right), j=1, \ldots, q$, are linearly independent, this linear independence is called a regularity condition (or constraint qualification).

Definition 4 (regular point see [25]). Let $I^{*}=\left\{i \mid g_{i}\left(x^{*}\right)=\right.$ 0 \}. Then $x^{*}$ is called a regular point of the constraints if the gradient vectors $\nabla g_{i}\left(x^{*}\right), \nabla h_{j}\left(x^{*}\right), i \in I^{*}, j=1, \ldots, q$, are linearly independent.

Lemma 5 (KKT theorem see [25]). In MP above, suppose that the objective function $f$ and the constraint functions $\mathbf{g}=$ $\left(g_{1}, \ldots, g_{p}\right)^{\prime}, \mathbf{h}=\left(h_{1}, \ldots, h_{q}\right)^{\prime}$ are continuously differentiable at a point $x^{*}$. If $x^{*}$ is a local minimum that satisfies some regularity conditions, then there exist a vector $\lambda \geq 0$ in $R^{m}$ and a vector $\boldsymbol{\mu}$ in $R^{n}$, called KKT multipliers, such that

$$
\nabla_{x} L\left(x^{*}, \lambda^{*}, \mu^{*}\right)=\mathbf{0}
$$

$$
\lambda^{\prime} \mathbf{g}\left(x^{*}\right)=0(\text { complementary slackness condition })
$$

where the Lagrangian function $L(x, \lambda, \mu)=f(x)+\lambda^{\prime} \mathbf{g}+\mu^{\prime} \mathbf{h}(x)$. 
Lemma 6 (see [26]). Let a matrix $M \in R^{m \times n}$ be given, matrix $M^{\dagger} \in R^{n \times m}$ which is called the Moore-Penrose pseudoinverse of $M$, such that

$$
\begin{array}{cc}
M M^{\dagger} M=M, & M^{\dagger} M M^{\dagger}=M^{\dagger}, \\
\left(M M^{\dagger}\right)^{\prime}=M M^{\dagger}, & \left(M^{\dagger} M\right)^{\prime}=M^{\dagger} M .
\end{array}
$$

Lemma 7 (see [26]). Let a symmetric matrix $M$ be given. Then

$$
\begin{array}{r}
M^{\prime^{\dagger}}=M^{\dagger^{\prime}}, \quad M M^{\dagger}=M^{\dagger} M, \quad M \geq 0 \\
\text { iff } M^{\dagger} \geq 0 .
\end{array}
$$

Lemma 8 ((extended Schur's lemma) see [27]). Let matrices $M=M^{\prime}, N$, and $R=R^{\prime}$ be given with appropriate sizes. Then the following conditions are equivalent:

(i) $M-N R^{\dagger} N^{\prime} \geq 0, R \geq 0$, and $N\left(I-R R^{\dagger}\right)=0$;

(ii) $\left[\begin{array}{cc}M & N \\ N^{\prime} & R\end{array}\right] \geq 0$;

(iii) $\left[\begin{array}{ll}R & N^{\prime} \\ N & M\end{array}\right] \geq 0$.

Lemma 9 (see [15]). Let matrices $L, M$, and $N$ be given. Then the matrix equation $L X M=N$ has a solution $X$ if and only if $L L^{\dagger} N M M^{\dagger}=N . X$ is given by $X=L^{\dagger} N M^{\dagger}+Y-L^{\dagger} L Y M M^{\dagger}$, where $Y$ is a matrix with an appropriate dimension.

Lemma 10 (see [15]). Let matrices $F=F^{\prime}, H$, and $G=G^{\prime}$ be given with appropriate sizes. Consider the following quadratic form:

$$
\begin{aligned}
& q(x, u) \\
& =E\left[x^{\prime}(t) F(t) x(t)+2 x^{\prime}(t) H(t) u(t)+u^{\prime}(t) G(t) u(t)\right],
\end{aligned}
$$

where $x$ and $u$ are random variables defined on a probability space $(\Omega, \mathfrak{B}, P)$. Then the following conditions are equivalent:

(i) $\inf _{u(t)} q(x, u)>-\infty$ for any random variable $x$;

(ii) there exists a symmetric matrix $W=W^{\prime}$ such that $\inf _{u(t)} q(x, u)=E\left[x^{\prime} W x\right]$ for any random variable $x$;

(iii) $G \geq O$ and $H\left(I-G G^{\dagger}\right)=0$;

(iv) $G \geq O$ and $\operatorname{Ker}(G) \subseteq \operatorname{Ker}(H)$;

(v) there exists a symmetric matrix $T=T^{\prime}$ such that $\left[\begin{array}{cc}F-T & H \\ H^{\prime} & G\end{array}\right] \geq 0$.

Moreover, if any of the above condition holds, then (ii) is satisfied by $W=F-H G^{\dagger} H^{\prime}$. In addition, $W \geq T$ for any $T$ satisfying $(v)$. Finally, for any random variable $x$, the random variable $u^{*}=-G^{\dagger} H^{\prime} x$ is optimal with the following optimal value:

$$
q\left(x, u^{*}\right)=E\left[x^{\prime}\left(F-H G^{\dagger} H^{\prime}\right) x\right]
$$

\section{Well-Posedness and Attainability under State Feedback Control}

In this section, we transform the LQ problem into an equivalent deterministic optimization problem. By means of the KKT theorem, we present a generalized difference Riccati equation (GDRE) without any positiveness constraint. Then, it is shown that the well-posedness and the attainability are equivalent to the solvability of GDRE.

Theorem 11. If the LQ optimal control problem (1)-(3) is attainable by $u(k)=K(k) x(k)$ and the regular point $\left(u_{*}(k), x_{*}(k)\right)$ is a locally optimal solution of problem (1)(3), then the following generalized difference Riccati equation (GDRE) has solutions $(P(k), \mu)$ with $0 \leq \mu \in R^{1}, k \in N_{T}$ :

$$
\begin{gathered}
P(k)=A^{\prime}(k) P(k+1) A(k)+C^{\prime}(k) P(k+1) C(k) \\
+Q(k)-H^{\prime}(k) G^{\dagger}(k) H(k), \\
H(k)=B^{\prime}(k) P(k+1) A(k) \\
\quad+\rho^{x u}(k) D^{\prime}(k) P(k+1) C(k), \\
G(k)=R(k)+B^{\prime}(k) P(k+1) B(k) \\
\quad+D^{\prime}(k) P(k+1) D(k) \geq \mathbf{0}, \quad k \in N_{T}, \\
\mu=\frac{\operatorname{tr}[P(T)-S]}{\operatorname{tr}\left(N^{\prime} N\right)}, \\
\boldsymbol{\mu}\left[\operatorname{tr}\left(X(T) N^{\prime} N\right)-\operatorname{tr}(M)\right]=0, \quad M=E\left[\xi \xi^{\prime}\right] .
\end{gathered}
$$

In addition,

$$
\begin{array}{r}
u_{*}(k)=\left[-G^{\dagger}(k) H(k)+Y(k)-G^{\dagger}(k) G(k) Y(k)\right] x(k), \\
Y(k) \in R^{m \times n}, \quad k \in N_{T}, \\
V\left(x_{0}\right)=\sum_{k=0}^{T-1} \operatorname{tr}[V(k) P(k+1)]+x_{0}^{\prime} P(0) x_{0}-\mu \operatorname{tr}(M) .
\end{array}
$$

Proof. Let $X(k)=E\left[x(k) x^{\prime}(k)\right]$ and $u(k)=K(k) x(k)$ for any $k \in N_{T}$; it can be shown that the LQ problem (1)-(3) can be rewritten as the following optimization problem:

$$
\begin{array}{ll}
\min _{K(0), \ldots, K(T-1)} \sum_{k=0}^{T-1} \operatorname{tr}\left[\left(Q(k)+K^{\prime}(k) R(k) K(k)\right) X(k)\right] \\
& +\operatorname{tr}[S X(T)] \\
\text { s.t. } \quad X(k+1) & \\
= & A(k) X(k) A^{\prime}(k)+C(k) X(k) C^{\prime}(k) \\
& +B(k) K(k) X(k) K^{\prime}(k) B^{\prime}(k) X(k) \\
& \times K^{\prime}(k) D^{\prime}(k) \\
& +A(k) X(k) K^{\prime}(k) B^{\prime}(k)
\end{array}
$$




$$
\begin{aligned}
&+B(k) K(k) X(k) A^{\prime}(k) \\
&+\rho^{x u}(k) D(k) K(k) X(k) C^{\prime}(k) \\
&+\rho^{x u}(k) C(k) X(k) K^{\prime}(k) D^{\prime}(k)+V(k), \\
& X(0)=E\left[x(0) x^{\prime}(0)\right]=x_{0} x_{0}^{\prime} \\
& \operatorname{tr}\left[X(T) N^{\prime} N\right] \leq \operatorname{tr} M, \quad M=E\left[\xi \xi^{\prime}\right] .
\end{aligned}
$$

Obviously, the problem (14) is a MP problem indicated as

$$
\begin{array}{ll}
\min & f[X(k), K(k)], \quad k \in N_{T}, \\
\text { s.t. } & h[X(k), K(k)]=0, \quad k \in N_{T}, \\
& g[X(T)] \leq 0,
\end{array}
$$

where

$$
\begin{aligned}
& f[X(k), K(k)] \\
& =\sum_{k=0}^{T-1} \operatorname{tr}\left[\left(Q(k)+K^{\prime}(k) R(k) K(k)\right) X(k)\right]+\operatorname{tr}[S X(T)], \\
& h[X(k), K(k)] \\
& =A(k) X(k) A^{\prime}(k)+C(k) X(k) C^{\prime}(k) \\
& \quad+B(k) K(k) X(k) K^{\prime}(k) B^{\prime}(k) X(k) K^{\prime}(k) D^{\prime}(k) \\
& \quad+A(k) X(k) K^{\prime}(k) B^{\prime}(k)+B(k) K(k) X(k) A^{\prime}(k) \\
& \quad+\rho^{x u}(k) D(k) K(k) X(k) C^{\prime}(k) \\
& \quad+\rho^{x u}(k) C(k) X(k) K^{\prime}(k) D^{\prime}(k)+V(k), \\
& g[X(T)]=\operatorname{tr}\left[X(T) N^{\prime} N\right]-M .
\end{aligned}
$$

According to KKT theorem, the Lagrangian function is defined as

$$
\begin{aligned}
& L(X(k), K(k), P(k+1), \mu) \\
& \quad=f[X(k), K(k)]+\sum_{k=0}^{T-1} \operatorname{tr}\{P(k+1) h[X(k), K(k)]\} \\
& \quad+\mu g[X(T)],
\end{aligned}
$$

where $\mu \in R^{1}$ and the matrices $P(0), P(1), \ldots, P(T)$ are Lagrangian multipliers.

Moreover, the following result,

$$
\frac{\partial L}{\partial X(k)}=\frac{\partial L}{\partial K(k)}=\frac{\partial L}{\partial X(T)}=0,
$$

is obvious.
By calculating, we conclude that $P(k)$ and $\lambda$ satisfy the equations of the form

$$
\begin{aligned}
{[R(k)} & \left.+B^{\prime}(k) P(k+1) B(k)+D^{\prime}(k) P(k+1) D(k)\right] K(k) \\
= & -B^{\prime}(k) P(k+1) A(k) \\
& -\rho^{x u}(k) D^{\prime}(k) P(k+1) C(k),
\end{aligned}
$$

$$
\begin{gathered}
P(k)=C^{\prime}(k) P(k) C(k) \\
+K^{\prime}(k)\left[R(k)+B^{\prime}(k) P(k+1) B(k)\right. \\
\left.+D^{\prime}(k) P(k+1) D(k)\right] K(k) \\
+\left[A^{\prime}(k) P(k+1) B(k)\right. \\
\left.+\rho^{x u}(k) D^{\prime}(k) P(k+1) C(k)\right] \\
+Q(k)+A^{\prime}(k) P(k+1) A(k), \\
P(T)=S+\mu N^{\prime} N .
\end{gathered}
$$

From Lemma 9, (19) has a solution $K(k)$ if and only if $G^{\dagger}(k) G(k) H(k)=H(k)$ and $K(k)=-G^{\dagger}(k) H(k)+Y(k)-$ $G^{\dagger}(k) G(k) Y(k)$, where

$$
\begin{array}{r}
G(k) \\
\quad=R(k)+B^{\prime}(k) P(k+1) B(k)+D^{\prime}(k) P(k+1) D(k), \\
H(k) \\
=B^{\prime}(k) P(k+1) A(k)+\rho^{x u}(k) D^{\prime}(k) P(k+1) C(k), \\
Y(k) \in R^{m \times n} .
\end{array}
$$

We substitute the above gains into (21); then the corresponding equations are formed as

$$
\begin{gathered}
P(k)=A^{\prime}(k) P(k+1) A(k)+C^{\prime}(k) P(k+1) C(k) \\
+Q(k)-H^{\prime}(k) G^{\dagger}(k) H(k), \\
H(k)=B^{\prime}(k) P(k+1) A(k) \\
\quad+\rho^{x u}(k) D^{\prime}(k) P(k+1) C(k), \\
G(k)=R(k)+B^{\prime}(k) P(k+1) B(k) \\
\quad+D^{\prime}(k) P(k+1) D(k) \geq 0, \\
\quad k \in N_{T}, \\
\mu=\frac{\operatorname{tr}[P(T)-S]}{\operatorname{tr}\left(N^{\prime} N\right)} .
\end{gathered}
$$


The only thing to note is that we can assume $P(k)$ is symmetric. Otherwise, we take $\widetilde{P}(k)=\left(P^{\prime}(k)+P(k)\right) / 2$.

Now we add the equality

$$
\begin{aligned}
& \sum_{k=0}^{T-1} E\left[\left(x^{\prime}(k+1) P(k+1) x(k+1)-x^{\prime}(k) P(k) x(k)\right)\right] \\
& \quad=E\left[x^{\prime}(T) P(T) x(T)-x_{0}^{\prime} P(0) x_{0}\right]
\end{aligned}
$$

to (2) and use (23); then we have

$$
\begin{aligned}
& J\left(x_{0}, u(0), \ldots, u(T-1)\right) \\
& =\sum_{k=0}^{T-1} E\left[\left(x^{\prime}(k) Q(k) x(k)+u^{\prime}(k) R(k) u(k)\right.\right. \\
& +x^{\prime}(k+1) P(k+1) x(k+1) \\
& \left.\left.\quad-x^{\prime}(k) P(k) x(k)\right)\right] \\
& +E\left[x^{\prime}(T)(S-P(T)) x(T)+x_{0}^{\prime} P(0) x_{0}\right] \\
& =\sum_{k=0}^{T-1} E\left[x ^ { \prime } ( k ) \left(Q(k)-P(k)+A^{\prime}(k) P(k+1) A(k)\right.\right. \\
& \left.+C^{\prime}(k) P(k+1) C(k)\right) x(k) \\
& +2 x^{\prime}(k)\left(A^{\prime}(k) P(k+1) B(k)\right. \\
& \left.+\rho^{x u}(k) C^{\prime}(k) P(k+1) D(k)\right) u(k) \\
& +u^{\prime}(k)\left(R(k)+B^{\prime}(k) P(k+1) B(k)\right. \\
& \left.\left.+D^{\prime}(k) P(k) D(k)\right) u(k)\right] \\
& +\sum_{k=0}^{T-1} E\left[\omega^{\prime}(k) P(k+1) \omega(k)\right] \\
& +E\left[x^{\prime}(T)(S-P(T)) x(T)+x_{0}^{\prime} P(0) x(0)\right] .
\end{aligned}
$$

By completion of square, we obtain

$$
\begin{aligned}
& J\left(x_{0}, u(0), \ldots, u(T-1)\right) \\
& =\sum_{k=0}^{T-1} E\left[\left(u(k)+G^{\dagger}(k) H(k) x(k)\right)^{\prime} G(k)\right. \\
& \left.\quad \times\left(u(k)+G^{\dagger}(k) H(k) x(k)\right)\right] \\
& +\sum_{k=0}^{T-1} \operatorname{tr}[V(k) P(k+1)] \\
& +E\left[x^{\prime}(T)(S-P(T)) x(T)\right]+x_{0}^{\prime} P(0) x_{0} .
\end{aligned}
$$

Here, we must prove that $G(k) \geq 0, k \in N_{T}$. Let us assume that there exists a $G(l)$ with a negative eigenvalue $\lambda$. Let $v_{\lambda}$ be the unitary eigenvector about $\lambda$; it implies that $v_{\lambda}^{\prime} v_{\lambda}=1$ and $G(l) v_{\lambda}=\lambda v_{\lambda}$. For any $\delta \neq 0$, let us suppose that a control sequence is given by

$$
\tilde{u}(k)= \begin{cases}-G\left({ }^{\dagger} k\right) H(k) x(k), & k \neq l, \\ \delta|\lambda|^{-1 / 2} v_{\lambda}-G^{\dagger}(k) H(k) x(k), & k=l .\end{cases}
$$

The corresponding cost is

$$
\begin{aligned}
J\left(x_{0}, \tilde{u}(0), \ldots, \tilde{u}(T-1)\right) & \\
= & \sum_{k=0}^{T-1} E\left[\left(u(k)+G^{\dagger}(k) H(k) x(k)\right)^{\prime} G(k)\right. \\
& \left.\times\left(u(k)+G^{\dagger}(k) H(k) x(k)\right)\right] \\
& +\sum_{k=0}^{T-1} \operatorname{tr}[V(k) P(k+1)] \\
& +E\left[x^{\prime}(T)(S-P(T)) x(T)\right]+x_{0}^{\prime} P(0) x_{0} \\
= & \left(\delta|\lambda|^{-1 / 2} v_{\lambda}\right)^{\prime} G(l)\left(\delta|\lambda|^{-1 / 2} v_{\lambda}\right) \\
& +\sum_{k=0}^{T-1} \operatorname{tr}[V(k) P(k+1)] \\
& +E\left[x^{\prime}(T)(S-P(T)) x(T)\right]+x_{0}^{\prime} P(0) x_{0} \\
= & -\delta^{2}+\sum_{k=0}^{T-1} \operatorname{tr}[V(k) P(k+1)] \\
& +E\left[x^{\prime}(T)(S-P(T)) x(T)\right]+x_{0}^{\prime} P(0) x_{0} .
\end{aligned}
$$

Letting $\delta \rightarrow \infty$, it yields $J\left(x_{0}, \widetilde{u}(0), \ldots, \widetilde{u}(T-1)\right) \rightarrow-\infty$, which is in contradiction with the attainability of the LQ problem (1)-(3).

From the above discussion and (21), it can be seen that the optimal value $V\left(x_{0}\right)$ is given by

$$
\begin{aligned}
V\left(x_{0}\right) & =J\left(x_{0}, u_{*}(0), \ldots, u_{*}(T-1)\right) \\
& =\sum_{k=0}^{T-1} \operatorname{tr}[V(k) P(k+1)]+x_{0}^{\prime} P(0) x_{0}-\mu \operatorname{tr}(M) .
\end{aligned}
$$

This proof is complete.

The following corollary shows that when $G(k)>0$ in GDRE (12), then $P(k), \mu$, and $u_{*}(k)$ are all unique.

Corollary 12. If the LQ optimal control problem (1)-(3) is attainable by $u(k)=K(k) x(k)$ and the regular point $\left(u_{*}(k), x_{*}(k)\right)$ is a locally optimal solution of problem (1)-(3), 
then the following GDRE has unique solutions $(P(k), \mu)$ with $0 \leq \mu \in R^{1}, k \in N_{T}$ :

$$
\begin{aligned}
& P(k)= A^{\prime}(k) P(k+1) A(k)+C^{\prime}(k) P(k+1) C(k) \\
&+ Q(k)-H^{\prime}(k) G^{-1}(k) H(k), \\
& H(k)= B^{\prime}(k) P(k+1) A(k) \\
&+\rho^{x u}(k) D^{\prime}(k) P(k+1) C(k), \\
& G(k)= R(k)+B^{\prime}(k) P(k+1) B(k) \\
&+D^{\prime}(k) P(k+1) D(k)>\mathbf{0}, \\
& \quad k \in N_{T}, \\
& \mu \frac{\operatorname{tr}[P(T)-S]}{\operatorname{tr}\left(N^{\prime} N\right)}, \\
& \boldsymbol{\mu}\left[\operatorname{tr}\left(X(T) N^{\prime} N\right)-\operatorname{tr} M\right]=0, \quad N=E\left[\xi \xi^{\prime}\right] .
\end{aligned}
$$

In addition,

$$
\begin{gathered}
u_{*}(k)=-G^{-1}(k) H(k) x(k), \quad k \in N_{T}, \\
V\left(x_{0}\right)=\sum_{k=0}^{T-1} \operatorname{tr}[V(k) P(k+1)]+x_{0}^{\prime} P(0) x_{0}-\mu \operatorname{tr} M .
\end{gathered}
$$

The following result is useful in the sequel, which gives an equivalent connection between the solvability of the GDRE and the well-posedness of the LQ problem.

Theorem 13. The LQ problem (1)-(3) is well posed; then there exist solutions $(P(k), \mu)$ to the GDRE (12). Conversely, if the GDRE (12) has solutions $(P(k), \mu)$, then the LQ problem (1)(3) is well posed. Moreover, the optimal cost satisfies

$$
V\left(x_{0}\right)=\sum_{k=0}^{T-1} \operatorname{tr}[V(k) P(k+1)]+x_{0}^{\prime} P(0) x_{0}-\mu \operatorname{tr}(M) .
$$

Proof. Necessity part: consider the following cost from $l$ to $T$ :

$$
\begin{aligned}
& W^{l}(x(l)) \\
& =\inf _{u(0), \ldots, u(T-1)} \sum_{k=l}^{T-1} E\left[\left(x^{\prime}(k) Q(k) x(k)\right.\right. \\
& \\
& \left.\left.\quad+u^{\prime}(k) R(k) u(k)\right)+x^{\prime}(T) S x(T)\right] .
\end{aligned}
$$

According to the optimal principle, if $W^{l}(x(l))$ is finite, so is $W^{m}(x(m))$ for any $l \leq m$. As $W^{0}\left(x_{0}\right)$ is finite, we can infer that $W^{l}(x(l))$ is finite for any $0 \leq l \leq T-1$.
Let $l=T-1$ and $P(T)=S+\mu N^{\prime} N$. By (1) and (33), it follows that

$$
\begin{aligned}
& W^{T-1}(x(T-1))-\operatorname{tr}[V(T-1) P(T)] \\
& -E\left[x^{\prime}(T)(S-P(T)) x(T)\right] \\
& =\inf _{u(T-1)} E\left[x^{\prime}(T-1) Q(T-1) x(T-1)\right. \\
& +u^{\prime}(T-1) R(T-1) u(T-1) \\
& \left.+x^{\prime}(T) S x(T)\right] \\
& -\operatorname{tr}[V(T-1) P(T)]-E\left[x^{\prime}(T)(S-P(T)) x(T)\right] \\
& =\inf _{u(T-1)} E\left[x^{\prime}(T-1)\right. \\
& \times\left(Q(T-1)+A^{\prime}(T-1) P(T) A(T-1)\right. \\
& \left.+C^{\prime}(T-1) P(T) C(T-1)\right) x(T-1) \\
& +2 x^{\prime}(T-1)\left(A^{\prime}(T-1) P(T) B(T-1)\right. \\
& +\rho^{x u}(T-1) C^{\prime} \\
& \times(T-1) P(T) D(T-1)) \\
& \times u(T-1) \\
& +u^{\prime}(T-1)(R(T-1) \\
& +B^{\prime}(T-1) P(T) B(T-1) \\
& \left.+D^{\prime}(T-1) P(T) D(T-1)\right) \\
& \times u(T-1)] \text {. }
\end{aligned}
$$

Applying Lemma 10 to the above quadratic form, there exists a symmetric matrix $P(T-1)$ such that

$$
\begin{aligned}
& W^{T-1}(x(T-1))-\operatorname{tr}[V(T-1) P(T)] \\
& -E\left[x^{\prime}(T)(S-P(T)) x(T)\right] \\
& =E\left[x^{\prime}(T-1) P(T-1) x(T-1)\right], \\
& P(T-1)=Q(T-1)+A^{\prime}(T-1) P(T) A(T-1) \\
& +C^{\prime}(T-1) P(T) C(T-1) \\
& -H^{\prime}(T-1) G^{\dagger}(T-1) H(T-1),
\end{aligned}
$$




$$
\begin{aligned}
& G(T-1)=R(T-1)+B^{\prime}(T-1) P(T) B(T-1) \\
& +D^{\prime}(T-1) P(T) D(T-1) \geq 0, \\
& H(T-1)=B^{\prime}(T-1) P(T) A(T-1) \\
& +\rho^{x u}(T-1) D^{\prime}(T-1) P(T) C(T-1) . \\
& W^{l}(x(l))-\operatorname{tr}[V(l) P(l+1)]-E\left[x^{\prime}(T)(S-P(T)) x(T)\right] \\
& =E\left[x^{\prime}(l) P(l) x(l)\right] \text {. }
\end{aligned}
$$

From (33), we have

$$
\begin{aligned}
& W^{l-1}(x(l-1))-\sum_{k=l-1}^{T-1} \operatorname{tr}[V(k) P(k+1)] \\
& -E\left[x^{\prime}(T)(S-P(T)) x(T)\right] \\
& =\inf _{u(l-1)} E\left[x^{\prime}(l-1) Q(l-1) x(l-1)\right. \\
& \left.\quad+u^{\prime}(l-1) R(l-1) u(l-1)+V^{l}(x(l))\right] \\
& \quad-\sum_{k=l-1}^{T-1} \operatorname{tr}[V(k) P(k+1)] \\
& -E\left[x^{\prime}(T)(S-P(T)) x(T)\right] \\
& =\inf _{u(l-1)} E\left[x^{\prime}(l-1) Q(l-1) x(l-1)\right. \\
& \left.\quad+u^{\prime}(l-1) R(l-1) u(l-1)+x^{\prime}(l) P(l) x(l)\right] \\
& \quad-\operatorname{tr}[V(l-1) P(l+1)]-E\left[x^{\prime}(T)(S-P(T)) x(T)\right] \\
& =\inf _{u(l-1)} E\left[x^{\prime}(l-1)(Q(l-1)\right. \\
& \quad+A^{\prime}(l-1) P(l-1) A(l-1) \\
& \left.+C^{\prime}(l-1) P(l-1) C(l-1)\right)
\end{aligned}
$$

$$
\begin{aligned}
& \times x(l-1) \\
& +2 x^{\prime}(l-1)\left(A^{\prime}(l-1) P(l) B(l-1)\right. \\
& +\rho^{x u}(l-1) C^{\prime}(l-1) \\
& \quad \times Q(l) D(l-1))
\end{aligned}
$$$$
\times u(l-1)
$$

$$
\begin{aligned}
& +u^{\prime}(l-1)\left(R(l-1)+B^{\prime}(l-1) P(l) B(l-1)\right. \\
& \left.+D^{\prime}(l-1) P(l) D(l-1)\right) \\
& \times u(l-1)] .
\end{aligned}
$$

By Lemma 10, it is straightforward that the finiteness of $V^{l-1} x(l-1)$ is equivalent to the following:

$$
\begin{gathered}
P(l-1)=A^{\prime}(l-1) P(l) A(l-1)+C^{\prime}(l-1) P(l) C(l-1) \\
+Q(l-1)-H^{\prime}(l-1) G^{\dagger}(l-1) H(l-1), \\
H(l-1)=B^{\prime}(l-1) P(l) A(l-1) \\
\quad+\rho^{x u}(l-1) D^{\prime}(l-1) P(l) C(l-1), \\
G(l-1)=R(l-1)+B^{\prime}(l-1) P(l) B(l-1) \\
\quad+D^{\prime}(l-1) P(l) D(l-1) \geq \mathbf{0}, \quad k \in N_{T}, \\
G(l-1) G^{\dagger}(l-1) H(l-1)-H(l-1)=0 .
\end{gathered}
$$

Moreover, $W^{l-1}(x(l-1))=\sum_{k=l-1}^{T-1} \operatorname{tr}[V(k) P(k+1)]+$ $E\left[x^{\prime}(T)(S-P(T)) x(T)\right]+x^{\prime}(l) P(l) x(l)$.

Sufficiency part: let

$$
\begin{aligned}
L(P(k+1))= & A^{\prime}(k) P(k+1) A(k) \\
& -P(k)+C^{\prime}(k) P(k+1) C(k)+Q(k), \\
M(P(k+1))= & A^{\prime}(k) P(k+1) B(k) \\
& +\rho^{x u}(k) C^{\prime}(k) P(k+1) D(k), \\
N(P(k+1))= & R(k)+B^{\prime}(k) P(k+1) B(k) \\
& +D^{\prime}(k) P(k+1) D(k) .
\end{aligned}
$$

Assume $P(1), \ldots, P(T)$ satisfy

$$
\left[\begin{array}{cc}
L(P(k+1)) & M(P(k+1)) \\
M^{\prime}(P(k+1)) & N(P(k+1))
\end{array}\right] \geq 0,
$$

for $k=0,1, \ldots, T-1$ and $P(T) \leq S+\mu N^{\prime} N$. 
As in the preceding,

$$
\begin{gathered}
J\left(x_{0}, u(0), \ldots, u(T-1)\right) \\
=\sum_{k=0}^{T-1} E\left[\left(x^{\prime}(k) Q(k) x(k)\right.\right. \\
\left.\left.+u^{\prime}(k) R(k) u(k)\right)+x^{\prime}(T) S x(T)\right] \\
=\sum_{k=0}^{T-1} E\left[\left(x^{\prime}(k) Q(k) x(k)+u^{\prime}(k) R(k) u(k)\right.\right. \\
\quad+x^{\prime}(k+1) P(k+1) x(k+1) \\
\left.\left.\quad-x^{\prime}(k) P(k) x(k)\right)\right] \\
+E\left[x^{\prime}(T)(S-P(T)) x(T)+x_{0}^{\prime} P(0) x_{0}\right] \\
+\sum_{k=0}^{T-1} E\left[x ^ { \prime } ( k ) \left(Q(k)-P(k)+A^{\prime}(k) P(k+1) A(k)\right.\right. \\
+\sum_{k=0} \operatorname{tr}[V(k) P(k+1)] \\
\left.+x^{\prime}(T) P(k+1) C(k)\right) x(k) \\
+2 x^{\prime}(k)\left(A^{\prime}(k) P(k+1) B(k)\right. \\
\left.+\rho^{\prime}(k) C^{\prime}(k) P(k+1) D(k)\right) u(k)+B^{\prime}(k) P(k+1) B(k)
\end{gathered}
$$

By Lemma 8, we get that

$$
\begin{aligned}
& J\left(x_{0}, u(0), \ldots, u(T-1)\right) \\
& \quad \geq \sum_{k=0}^{T-1} \operatorname{tr}[V(k) P(k+1)]+x_{0}^{\prime} P(0) x(0)-\mu \operatorname{tr} M
\end{aligned}
$$

In other words,

$$
V\left(x_{0}\right)=\sum_{k=0}^{T-1} \operatorname{tr}[V(k) P(k+1)]+x_{0}^{\prime} P(0) x(0)-\mu \operatorname{tr} M,
$$

which implies that the LQ problem (1)-(3) is well posed.

We are now equipped to present the main result in this section.
Theorem 14. The following assertions are equivalent.

(i) The LQ problem (1)-(3) is attainable.

(ii) The LQ problem (1) - (3) is well posed.

(iii) The GDRE (12) is solvable.

In addition, the feedback control law is achieved by

$$
\begin{aligned}
& u(k)=-\left[R(k)+B^{\prime}(k) P(k+1) B(k)\right. \\
&\left.+D^{\prime}(k) P(k+1) D(k)\right]^{\dagger} \\
& \times {\left[B^{\prime}(k) P(k+1) A(k)+\rho^{x u}(k) D^{\prime}(k) P\right.} \\
&\times(k+1) C(k)] x(k),
\end{aligned}
$$

where $P(0), \ldots, P(T)$ are solutions to the GDRE (12) and $k \in$ $N_{T}$.

Proof. By Theorem 13, it is easy to have that (ii) is equivalent to (iii). Our objective is to show that (i) is equivalent to (iii). From Theorem 11, we only need to show (iii) $\Rightarrow$ (i).

Suppose the GDRE (12) admits a pair of solutions $(P(1), \ldots, P(T), \mu)$. By the same way as Theorem 11 , the following can be proved:

$$
\begin{aligned}
& J\left(x_{0}, u(0), \ldots, u(T-1)\right) \\
& =\sum_{k=0}^{T-1} E\left[\left(x^{\prime}(k) Q(k) x(k)+u^{\prime}(k) R(k) u(k)\right)\right. \\
& \left.+x^{\prime}(T) S x(T)\right] \\
& =\sum_{k=0}^{T-1} E\left[\left(x^{\prime}(k) Q(k) x(k)\right.\right. \\
& \quad+u^{\prime}(k) R(k) u(k) \\
& +x^{\prime}(k+1) P(k+1) x(k+1) \\
& \left.\left.\quad-x^{\prime}(k) P(k) x(k)\right)\right] \\
& +E\left[x^{\prime}(T)(S-P(T)) x(T)+x_{0}^{\prime} P(0) x_{0}\right] \\
& =\sum_{k=0}^{T-1} E\left[x^{\prime}(k)(Q(k)-P(k)\right. \\
& +A^{\prime}(k) P(k+1) A(k) \\
& \left.+C^{\prime}(k) P(k+1) C(k)\right) x(k)
\end{aligned}
$$




$$
\begin{gathered}
+2 x^{\prime}(k)\left(A^{\prime}(k) P(k+1) B(k)\right. \\
+\rho^{x u}(k) C^{\prime}(k) P \\
\times(k+1) D(k)) u(k) \\
+u^{\prime}(k)\left(R(k)+B^{\prime}(k) P(k+1) B(k)\right. \\
\left.\left.+D^{\prime}(k) P(k) D(k)\right) u(k)\right] \\
+\sum_{k=0}^{T-1} \operatorname{tr}[V(k) P(k+1)] \\
+E\left[x^{\prime}(T)(S-P(T)) x(T)+x_{0}^{\prime} P(0) x(0)\right] \\
=\sum_{k=0}^{T-1} E\left[\left(u(k)+G^{\dagger}(k) H(k) x(k)\right)^{\prime} G(k)\right. \\
\left.\times\left(u(k)+G^{\dagger}(k) H(k) x(k)\right)\right] \\
+\sum_{k=0}^{T-1} \operatorname{tr}[V(k) P(k+1)] \\
+E\left[x^{\prime}(T)(S-P(T)) x(T)\right]+x_{0}^{\prime} P(0) x_{0} .
\end{gathered}
$$

So, the optimal value $V\left(x_{0}\right)=\sum_{k=0}^{T-1} \operatorname{tr}[V(k) P(k+$ 1)] $+x_{0}^{\prime} P(0) x_{0}-\mu \operatorname{tr}(M)$ and the feedback $u(k)=$ $-G^{\dagger}(k) H(k) x(k)$.

\section{Relation between Optimal Synthesis and GDRE}

In this section, we first attempt to verify that any optimal control can be denoted by virtue of the solution of the GDRE (12) with two degrees of freedom and the optimal cost is given.

Theorem 15. Assume that the GDRE (12) admits a solution. Then the optimal control satisfies the following:

$$
\begin{aligned}
u^{Y(k), Z(k)}(k) & \\
= & -\left(G^{\dagger}(k) H(k)+Y(k)-G^{\dagger}(k) G(k) Y(k)\right) x(k) \\
& +Z(k)-G^{\dagger}(k) G(k) Z(k),
\end{aligned}
$$

where $Y(k), k \in N_{T}$ are arbitrary random variables defined on the probability space $\{\Omega, F, \mu\}$. And the optimal cost value is given by

$$
V\left(x_{0}\right)=\sum_{k=0}^{T-1} \operatorname{tr}[V(k) P(k+1)]+x_{0}^{\prime} P(0) x_{0}-\mu \operatorname{tr}(M),
$$

where $P(0), \ldots, P(T-1)$ solve the GDRE (12).
Proof. Suppose the GDRE (12) admits solutions $P(0), \ldots, P(T-1)$. As the preceding calculation, we have

$$
\begin{aligned}
& J\left(x_{0}, u(0), \ldots, u(T-1)\right) \\
& =\sum_{k=0}^{T-1} E\left[\left(x^{\prime}(k) Q(k) x(k)\right.\right. \\
& \left.\left.+u^{\prime}(k) R(k) u(k)\right)+x^{\prime}(T) S x(T)\right] \\
& =\sum_{k=0}^{T-1} E\left[\left(x^{\prime}(k) Q(k) x(k)\right.\right. \\
& +u^{\prime}(k) R(k) u(k) \\
& +x^{\prime}(k+1) P(k+1) x(k+1) \\
& \left.\left.-x^{\prime}(k) P(k) x(k)\right)\right] \\
& +E\left[x^{\prime}(T)(S-P(T)) x(T)+x_{0}^{\prime} P(0) x_{0}\right] \\
& =\sum_{k=0}^{T-1} E\left[x^{\prime}(k)(Q(k)-P(k)\right. \\
& +A^{\prime}(k) P(k+1) A(k) \\
& \left.+C^{\prime}(k) P(k+1) C(k)\right) x(k) \\
& +2 x^{\prime}(k)\left(A^{\prime}(k) P(k+1) B(k)\right. \\
& +\rho^{x u}(k) C^{\prime}(k) P \\
& \times(k+1) D(k)) u(k) \\
& +u^{\prime}(k)(R(k) \\
& +B^{\prime}(k) P(k+1) B(k) \\
& \left.\left.+D^{\prime}(k) P(k) D(k)\right) u(k)\right] \\
& +\sum_{k=0}^{T-1} \operatorname{tr}[V(k) P(k+1)] \\
& +E\left[x^{\prime}(T)(S-P(T)) x(T)+x_{0}^{\prime} P(0) x(0)\right] \\
& =\sum_{k=0}^{T-1} E\left[x^{\prime}(k) H^{\prime}(k) G^{\dagger}(k) H(k) x(k)\right. \\
& \left.+2 x^{\prime}(k) H^{\prime}(k) u(k)+u^{\prime}(k) G(k) u(k)\right] \\
& +\sum_{k=0}^{T-1} \operatorname{tr}[V(k) P(k+1)] \\
& +E\left[x^{\prime}(T)(S-P(T)) x(T)\right]+x_{0}^{\prime} P(0) x_{0} .
\end{aligned}
$$


Let $F_{1}(k)=G^{\dagger}(k) G(k) Y(k)-Y(k)$ and $F_{2}(k)=$ $G^{\dagger}(k) G(k) Z(k)-Z(k)$; then

$$
G(k) F_{1}(k)=G(k) F_{2}(k)=0 .
$$

So, $J\left(x_{0}, u(0), \ldots, u(T-1)\right)$ can be rewritten as

$$
\begin{aligned}
& J\left(x_{0}, u(0), \ldots, u(T-1)\right) \\
& =\sum_{k=0}^{T-1} E\left[\left(u(k)+\left(G^{\dagger}(k) H(k)+F_{1}(k)\right) x(k)\right.\right. \\
& \left.+F_{2}(k)\right)^{\prime} G(k) \\
& \times\left(u(k)+\left(G^{\dagger}(k) H(k)+F_{1}(k)\right) x(k)\right. \\
& \left.\left.+F_{2}(k)\right)\right] \\
& +\sum_{k=0}^{T-1} \operatorname{tr}[V(k) P(k+1)] \\
& +E\left[x^{\prime}(T)(S-P(T)) x(T)\right]+x_{0}^{\prime} P(0) x_{0} .
\end{aligned}
$$

Because of $G(k) \geq 0$, we immediately obtain that

$$
V\left(x_{0}\right)=\sum_{k=0}^{T-1} \operatorname{tr}[V(k) P(k+1)]+x_{0}^{\prime} P(0) x_{0}-\mu \operatorname{tr}(M),
$$

and the control $u(k)=-\left(G^{\dagger}(k) H(k)+F_{1}(k)\right) x(k)+F_{2}(k)$.

Now, we are interested in arbitrary control sequence $(\widetilde{u}(0), \ldots, \widetilde{u}(T-1))$ which minimizes the cost function $J$. So we deduce that

$$
\begin{aligned}
J\left(x_{0}, \tilde{u}(0), \ldots, \widetilde{u}(T-1)\right) \\
=\sum_{k=0}^{T-1} E\left[\left(\widetilde{u}(k)+G^{\dagger}(k) H(k) x(k)\right)^{\prime} G(k)\right. \\
\left.\quad \times\left(\widetilde{u}(k)+G^{\dagger}(k) H(k) x(k)\right)\right] \\
+\sum_{k=0}^{T-1} \operatorname{tr}[V(k) P(k+1)] \\
+E\left[x^{\prime}(T)(S-P(T)) x(T)\right]+x_{0}^{\prime} P(0) x_{0} \\
=\sum_{k=0}^{T-1} \operatorname{tr}[V(k) P(k+1)] \\
\quad+E\left[x^{\prime}(T)(S-P(T)) x(T)\right]+x_{0}^{\prime} P(0) x_{0} .
\end{aligned}
$$

Thus there must be

$$
\begin{aligned}
& E\left[\left(\widetilde{u}(k)+G^{\dagger}(k) H(k) x(k)\right)^{\prime} G(k)\right. \\
& \left.\quad \times\left(\widetilde{u}(k)+G^{\dagger}(k) H(k) x(k)\right)\right]=0 .
\end{aligned}
$$

By $G(k) \geq 0$, the above is equivalent to the following:

$$
G(k)\left(\widetilde{u}(k)+G^{\dagger}(k) H(k) x(k)\right)=0 .
$$

From Lemma 10, it follows that

$$
\widetilde{u}(k)=-G^{\dagger}(k) H(k) x(k)+Z(k)-G^{\dagger} G(k) Z(k) .
$$

The following numerical example illustrates the effectiveness of our theoretical results.

Example 16. The coefficients of the systems (1)-(2) are as follows:

$$
\begin{array}{cc}
A(0)=\left[\begin{array}{ll}
1 & 0 \\
0 & 2
\end{array}\right], & B(0)=\left[\begin{array}{l}
2 \\
1
\end{array}\right], \\
C(0)=\left[\begin{array}{cc}
-1 & 0 \\
0 & 1
\end{array}\right], & D(0)=\left[\begin{array}{c}
1 \\
-1
\end{array}\right], \\
V(0)=\left[\begin{array}{ll}
2 & 1 \\
1 & 1
\end{array}\right], & \rho^{x u}(0)=-2, \\
x(0)=\left[\begin{array}{ll}
1 & 2 \\
3 & 4
\end{array}\right], & Q(0)=\left[\begin{array}{c}
-1 \\
1
\end{array}\right], \\
S=\left[\begin{array}{ll}
1 & 0 \\
0 & 1
\end{array}\right], & R(0)=-13, \\
a_{11}=a_{22}=1, & a_{12}=a_{21}=0 \\
\xi_{1}=\xi_{2}=1 .
\end{array}
$$

We solve the corresponding GDRE (12) and calculate the optimal cost value:

$$
\begin{gathered}
P(1)=\left[\begin{array}{ll}
2 & 0 \\
0 & 2
\end{array}\right], \quad H(0)=\left[\begin{array}{ll}
8 & 8
\end{array}\right], \\
G(0)=1, \quad P(0)=\left[\begin{array}{ll}
-65 & -64 \\
-64 & -52
\end{array}\right], \\
\mu=1, \quad V\left(x_{0}\right)=17 .
\end{gathered}
$$

\section{Conclusion}

This paper mainly studies linear quadratic optimal control with inequality constraint for discrete-time indefinite stochastic systems. With the aid of the KKT theorem, we present a necessary and sufficient condition under which the problem is well posed and a state feedback control can be derived. Moreover, it is shown that the solvability of the GDRE, the well-posedness, and the attainability of the LQ problem are equivalent to each other. Finally, we give a structure of all optimal controls. To some degree, the previous results on stochastic LQ control without constraint can be regarded as corollaries of the main theorems of this paper.

\section{Conflict of Interests}

The authors declare that there is no conflict of interests regarding the publication of this paper. 


\section{Acknowledgments}

This work is supported by NSF of China (Grants nos. 61170054, 61174078, and 61402265), the Research Fund for the Taishan Scholar Project of Shandong Province of China, the SDUST Research Fund (Grant no. 2011KYTD105), and the State Key Laboratory of Alternate Electrical Power System with Renewable Energy Sources (Grant no. LAPS13018).

\section{References}

[1] R. E. Kalman, "Contributions to the theory of optimal control," Boletín de la Sociedad Matemática Mexicana, vol. 5, pp. 102-119, 1960.

[2] B. D. O. Anderson and J. B. Moore, Optimal Control Linear Quadratic Methods, Prentice-Hall, New York, NY, USA, 1989.

[3] F. L. Lewis, Optimal Control, John Wiley \& Sons, New York, NY, USA, 1986.

[4] W. M. Wonham, "On a matrix Riccati equation of stochastic control," SIAM Journal on Control and Optimization, vol. 6, pp. 681-697, 1968.

[5] M. Athans, "Special issue on the linear-quadratic-Gaussian estimation and control problem," IEEE Transactions on Automatic Control, vol. 16, pp. 527-547, 1971.

[6] A. Bensoussan, Stochastic Control of Partially Observable Systems, Cambridge University Press, Cambridge, UK, 1992.

[7] M. H. A. Davis, Linear Estimation and Stochastic Control, Chapman and Hall, London, UK, 1977.

[8] E. Yaz, "Infinite horizon quadratic optimal control of a class of nonlinear stochastic systems," IEEE Transactions on Automatic Control, vol. 34, no. 11, pp. 1176-1180, 1989.

[9] W. Zhang and B. Chen, "On stabilizability and exact observability of stochastic systems with their applications," Automatica, vol. 40, no. 1, pp. 87-94, 2004.

[10] W. Zhang, H. Zhang, and B. S. Chen, "Generalized Lyapunov equation approach to state-dependent stochastic stabilization/detectability criterion," IEEE Transactions on Automatic Control, vol. 53, no. 7, pp. 1630-1642, 2008.

[11] Y. Huang, W. Zhang, and H. Zhang, "Infinite horizon linear quadratic optimal control for discrete-time stochastic systems," Asian Journal of Control, vol. 10, no. 5, pp. 608-615, 2008.

[12] S. Chen, X. Li, and X. Y. Zhou, "Stochastic linear quadratic regulators with indefinite control weight costs," SIAM Journal on Control and Optimization, vol. 36, no. 5, pp. 1685-1702, 1998.

[13] R. T. Ku and M. Athans, "Further results on the uncertainty threshold principle," IEEE Transactions on Automatic Control, vol. 22, no. 5, pp. 866-868, 1977.

[14] A. Beghi and D. D’Alessandro, "Discrete-time optimal control with control-dependent noise and generalized Riccati difference equations," Automatica, vol. 34, no. 8, pp. 1031-1034, 1998.

[15] M. A. Rami, X. Chen, and X. Y. Zhou, "Discrete-time indefinite LQ control with state and control dependent noises," Journal of Global Optimization, vol. 23, no. 3-4, pp. 245-265, 2002.

[16] W. Zhang and B. Chen, " $H$-representation and applications to generalized Lyapunov equations and linear stochastic systems," IEEE Transactions on Automatic Control, vol. 57, no. 12, pp. 3009-3022, 2012.

[17] B. Chen and W. Zhang, "Stochastic $H_{2} / H_{\infty}$ control with statedependent noise," IEEE Transactions on Automatic Control, vol. 49, no. 1, pp. 45-57, 2004.
[18] T. Hou, W. Zhang, and H. Ma, "Finite horizon $H_{2} / H_{\infty}$ control for discrete-time stochastic systems with Markovian jumps and multiplicative noise," IEEE Transactions on Automatic Control, vol. 55, no. 5, pp. 1185-1191, 2010.

[19] Y. L. Huang and W. H. Zhang, "Study on stochastic linear quadratic optimal control with constraint," Acta Automatica Sinica, vol. 32, no. 2, pp. 246-254, 2006.

[20] X. Liu, Y. Li, and W. Zhang, "Stochastic linear quadratic optimal control with constraint for discrete-time systems," Applied Mathematics and Computation, vol. 228, pp. 264-270, 2014.

[21] G. Li and W. H. Zhang, "Discrete-time indefinite stochastic linear quadratic optimal control with equality constraints," in Proceedings of the 25th Chinese Control and Decision Conference (CCDC '13), pp. 4999-5004, May 2013.

[22] G. Li and W. Zhang, "Discrete-time indefinite stochastic linear quadratic optimal control: inequality constraint case," in Proceedings of the 32nd Chinese Control Conference (CCC '13), pp. 2327-2332, July 2013.

[23] H. Dong, Z. Wang, D. W. C. Ho, and H. Gao, "Varianceconstrained $H_{\infty}$ filtering for a class of nonlinear time-varying systems with multiple missing measurements: the finitehorizon case," IEEE Transactions on Signal Processing, vol. 58, no. 5, pp. 2534-2543, 2010.

[24] L. Ma, Y. Bo, Y. Zhou, and Z. Guo, "Error variance-constrained $H_{\infty}$ filtering for a class of nonlinear stochastic systems with degraded measurements: the finite horizon case," International Journal of Systems Science, vol. 43, no. 12, pp. 2361-2372, 2012.

[25] D. G. Luenberger, Optimization by Vector Space Methods, John Wiley \& Sons, New York, NY, USA, 1968.

[26] R. Penrose, "A generalized inverse of matrices," Cambridge Philosophical Society, vol. 57, pp. 17-19, 1955.

[27] A. Albert, "Conditions for positive and nonnegative definiteness in terms of pseudoinverses," SIAM Journal on Applied Mathematics, vol. 17, pp. 434-440, 1969. 


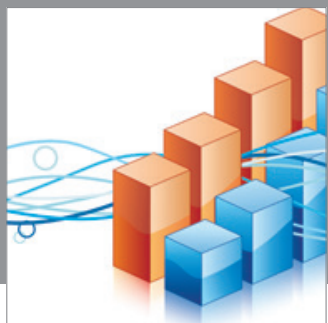

Advances in

Operations Research

mansans

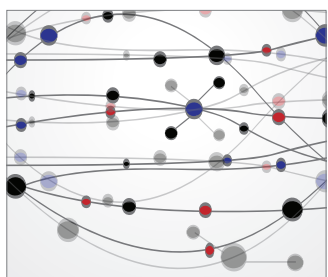

The Scientific World Journal
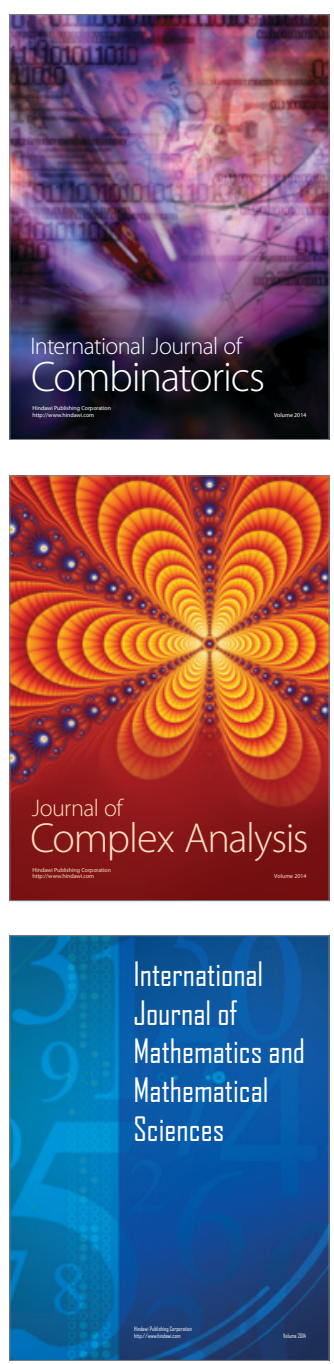
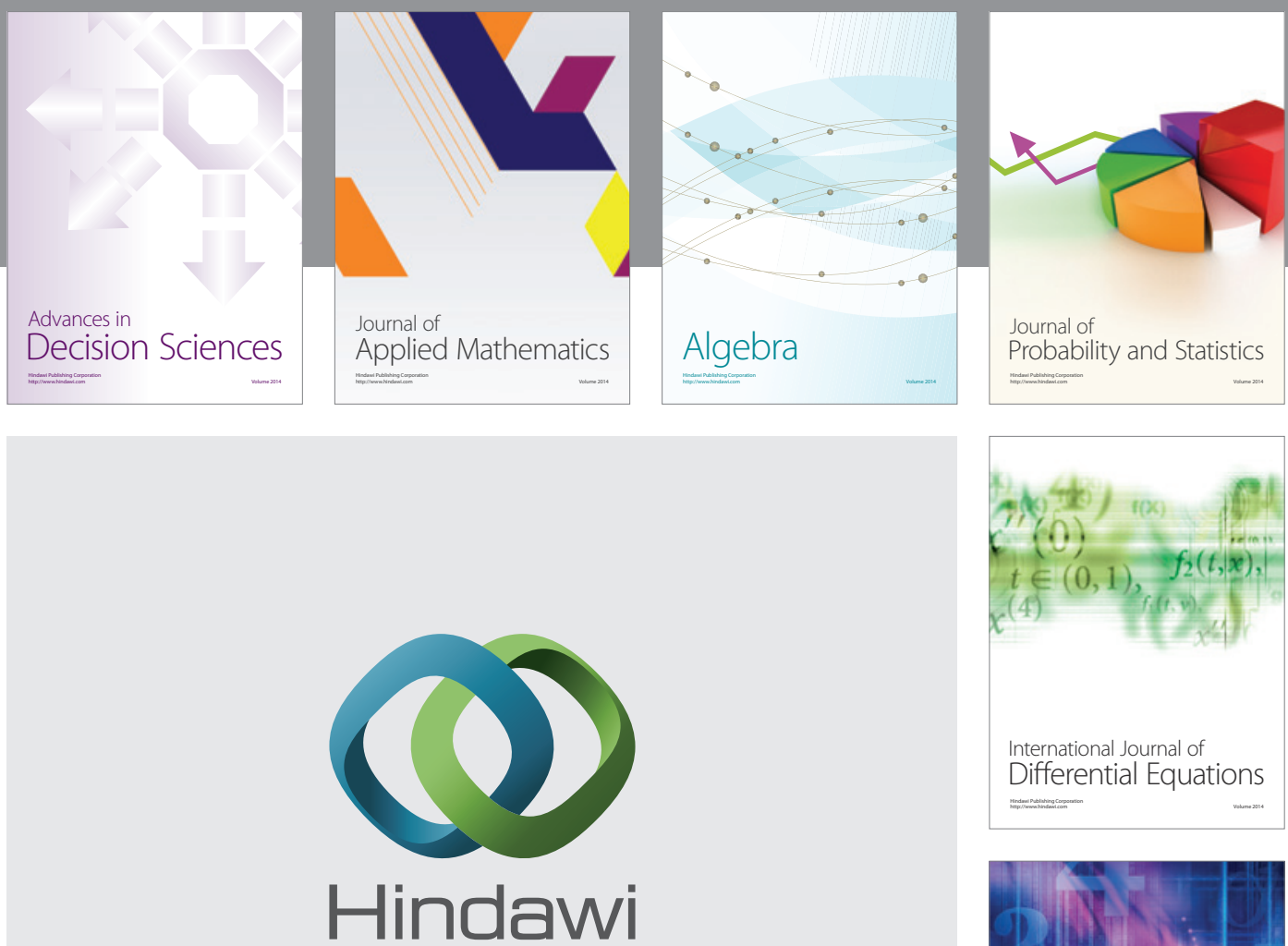

Submit your manuscripts at http://www.hindawi.com
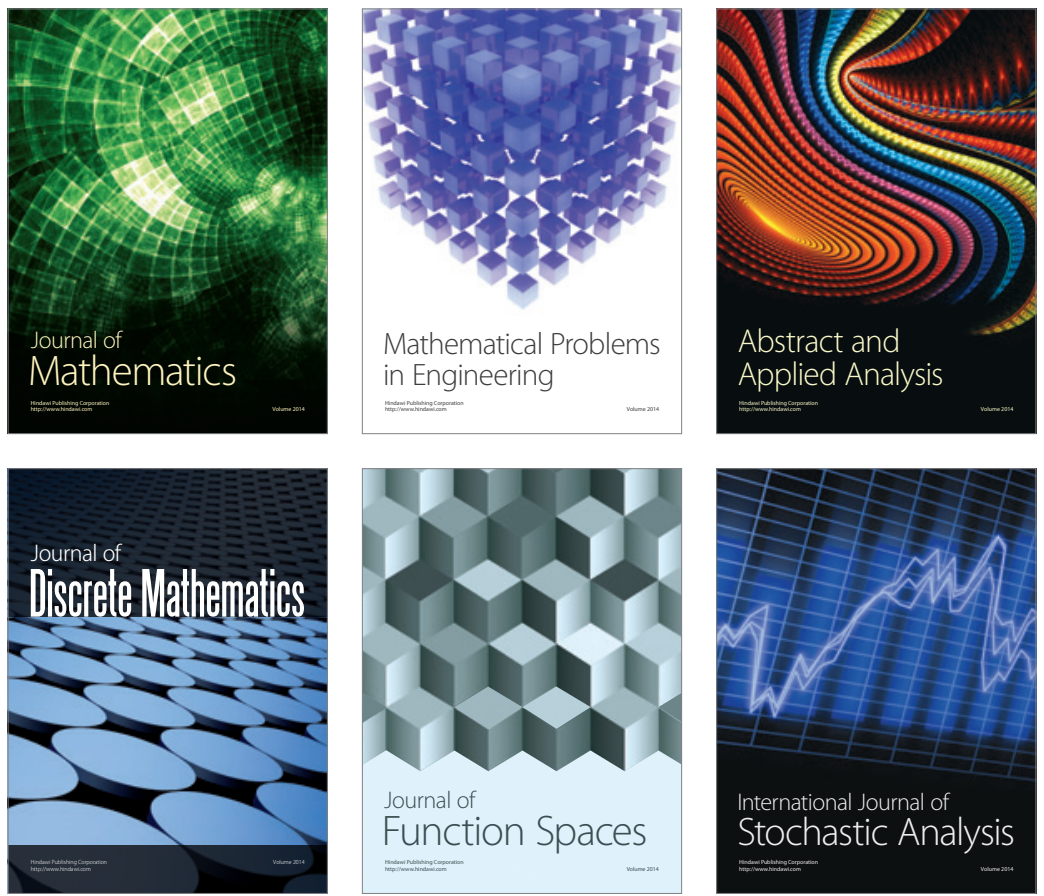

Journal of

Function Spaces

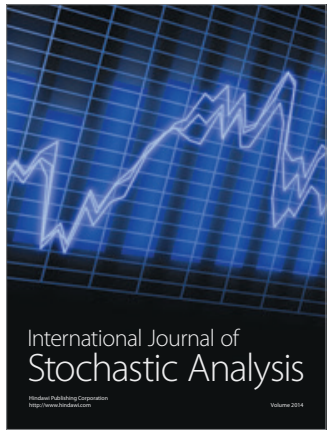

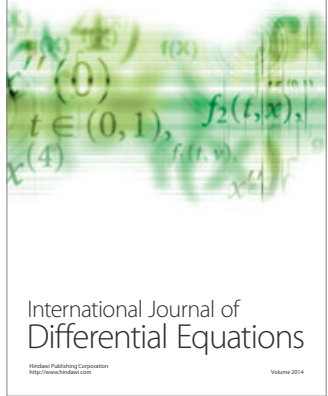
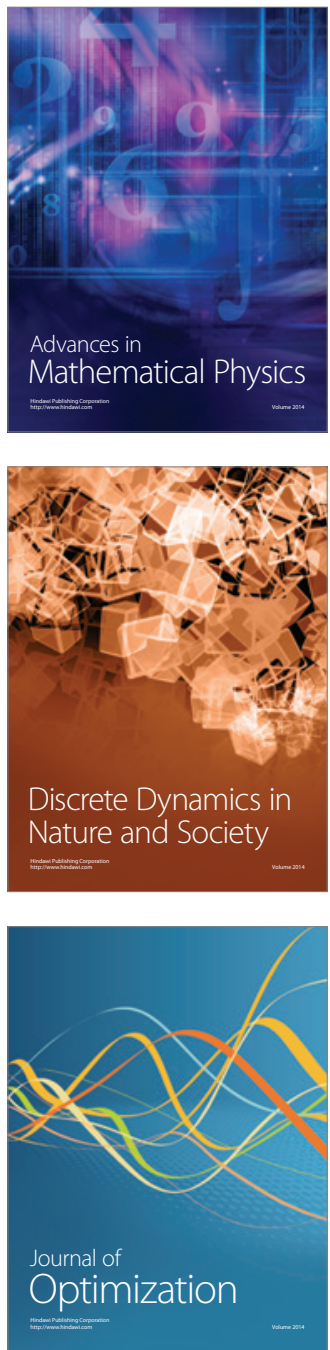